Alain Rabatel

IUFM de Lyon, UMR CNRS 5191

Université Lyon 2, ENS-LSH

alain.rabatel@lyon.iufm.fr

\title{
Le dialogisme du point de vue dans les comptes rendus de perception
}

On se propose d'analyser les relations que le point de vue (PDV), appréhendé à travers les comptes rendus de perception, entretient avec les paroles ou les pensées rapportées, selon une conception qui intègre le discours " rapporté $\mathrm{I}$ » à la problématique plus vaste du dialogisme (Bres et Verine 2002 : I 59).

Sur le plan sémantique, le compte rendu de perception correspond à la trace explicite ou implicite de savoirs, de jugements de valeur intervenant dans la référenciation des objets du discours à partir d'un procès perceptuel, et dans le modus et dans le dictum (cf. Ducrot I 993 : I 28). Comme la référenciation des objets y révèle le PDV de l'énonciateur, le compte rendu de perception fait voler en éclats l'opposition objectivité/subjectivité et correspond à une modalité d'expression de la subjectivité d'un sujet, complémentaire de celles qui apparaissent dans les comptes rendus de paroles ou de pensées, avec lesquels il peut se combiner. Sur le plan énonciatif, le compte rendu de perception, comme tout PDV, est pris en charge par le sujet de l'énonciation et/ou par un sujet interne à l'énoncé, en l'occurrence le sujet des perceptions (Rabatel I 998).

Notre premier objectif sera de baliser le champ des marques de la subjectivité en nous intéressant non au système personnel, largement exploré depuis Benveniste, mais à celui des marques que l'on range derrière les expressions de sujet modal ou d'actualisation modale, afin de mettre en relief le dialogisme des comptes rendus de perception. Le deuxième objectif sera de préciser comment l'actuali-

I. Ou « représenté » cf. Nølke et Olsen 2000:87 et Rabatel 2003a. 
sation modale des comptes rendus de perception objective les liens entre observable, pensable et dicible ${ }^{\mathrm{I}}$, alimentant une conception dynamique de la subjectivité. En effet, les degrés d'actualisation des comptes rendus de perception ne se contentent pas d'individualiser les objets perçus, ils participent à l'objectivation et à l'individualisation du sujet percevant, pensant et parlant. On verra en conclusion que cette conception émergente et graduelle de la subjectivité ${ }^{2}$ alimente - modestement - les hypothèses de la praxématique sur la dynamique de construction du sujet en idem/aliud et en ipse.

\section{Locuteur, énonciateur, actualisation déictique et actualisation modale}

Notre conception de l'énonciation emprunte au modèle énonciatif de Ducrot 1984, qui repose sur la distinction (complexe, ainsi que le rappellent Charaudeau et Maingueneau : 2002, 220-224, 226) entre locuteur et énonciateur, tout en s'en démarquant sur le fait que nous considérons que les PDV des énonciateurs ont une valeur d'acte de langage indirect, du fait de la valeur argumentative indirecte des PDV, ce que nous avons appelé l'effet-PDV. Le locuteur $(L)$ est l'instance qui profère un énoncé (dans ses dimensions phonétiques et phatique ou dans ses réalisations scripturales) selon un repérage déictique à partir d'un ego, hic et nunc ou selon un repérage indépendant de sa situation d'énonciation. L'énonciateur $(E 3)$ est à l'origine d'un PDV (Ducrot I 984 : 204) : proche du sujet modal de Bally, c'est l'instance des actualisations modales, ce qui implique

I. «Il n'y a pas la pensée et le langage, chacun des deux ordres à l'examen se dédouble et envoie un rameau dans l'autre [...] Les opérations expressives se passent entre parole pensante et pensée parlante, et non pas, comme on le dit légèrement, entre pensée et langage. Ce n'est pas parce qu'ils sont parallèles que nous parlons, c'est parce que nous parlons qu'ils sont parallèles » (Merleau-Ponty, Signes, Gallimard I960:26).

2. Abordée sous l'angle de la (pré)-réflexivité des perceptions dans Rabatel 200 Ia et d, 2003a, b, 2004b. Le présent article prolonge les analyses esquissées dans Rabatel $2003 \mathrm{~b}$.

3. On notera par une majuscule, suivie du chiffre I, ce locuteur primaire et cet énonciateur primaire, et par une barre oblique le syncrétisme de Li / EI. Les locuteurs et énonciateurs enchâssés (ou cités) dans l'énoncé du locuteur citant, et dans le point de vue originel à partir duquel se marquent les positions énonciatives divergentes seront notés par une minuscule suivie du chiffre 2,12 et e2. 
qu'elle assume l'énoncé, en un sens nettement moins abstrait que la prise en charge découlant de l'ancrage déictique. Une telle conception de l'énonciateur permet de rendre compte de la valeur pragmatique de PDV qui ne s'expriment pas dans des paroles, notamment en contexte d'effacement énonciatif (Vion 200I, Philippe 2002, Rabatel 2003a : 57-64).

C'est ainsi qu'en (I), pour reprendre un exemple célèbre de Ducrot, le PDV de l'énonciateur (e2) Jeanne, exprime un acte de langage expressif car l'énoncé qui suit mais ne se contente pas de dénoter un état du monde [/il pleut/ - voire /il continue de pleuvoir/], il indique le vif souhait de sortir [/je veux sortir (maintenant que mes valises sont prêtes)/] :

(I) Jeanne, ayant fini ses malles, s'approcha de la fenêtre, mais la pluie ne cessait pas (Maupassant, Une Vie, in Ducrot I980: 20).

Et c'est essentiellement la saisie de cet acte de langage indirect qui permet d'interpréter correctement l'énoncé sur la base d'une saturation sémantique du sujet modal. Car Jeanne ne se réduit pas à un seul foyer de perception, autrement dit à un pur centre scopique, elle est présentée ici, du fait même de la référenciation de sa perception, comme un sujet praxéologique : en ce sens, il est indéniable que l'évaluation de sa perception, dans son amont comme dans son aval, est partie intégrante d'un schéma actionnel, reposant sur des schèmes d'expérience pratique orientés vers un but - comme l'analyse de l'exemple (3) le confirmera.

Il est certain que la bipartition locuteur/énonciateur ne va pas de soi : nous ne visons pas ici le fait que ce que les uns appellent locuteur corresponde pour les autres à l'énonciateur, mais le fait qu'au-delà des différences de dénomination, la plupart des théoriciens soient d'accord, à l'instar de Guillemin-Flescher, (ou de Bres et Verine 2002 : I63, dans un autre champ théorique) pour considérer que l'actualisation modale aille de pair avec l'actualisation déictique :

L'énonciateur est ici envisagé comme point de référence abstrait dans le calcul du temps, de l'espace, et des personnes et comme support des modalités. Le locuteur correspond à celui qui prend 
la parole. Les deux peuvent coïncider, mais ce n'est pas nécessairement le cas : par exemple, dans le discours indirect libre (GuilleminFlescher 2003 : I 83).

Cette définition, conforme aux analyses culioliennes, mais encore au premier Benveniste, mêle critères relevant de la déixis et du sujet modal dans le marquage de l'énonciation personnelle, comme si l'actualisation déictique allait nécessairement de pair avec l'actualisation modale. Elle mentionne bien le cas possible de déliaison dans le DIL, mais sans revenir pour autant sur la coïncidence des actualisations, qui semble un trait définitoire, comme si le DIL était une réalisation discursive à la limite du système. Or, si l'on considère au contraire que le DIL ne fait que révéler la nature profondément dialogique du langage, alors, l'exception devient la règle.

Il est incontestable que le DIL ne fasse pas coïncider les deux actualisations (Rabatel 2003a : 57-58) :

(2) Edipe fit part de ses projets aux citoyens de Thèbes : il allait épouser cette malheureuse Jocaste.

En effet, « cette malheureuse » peut renvoyer autant au PDV d'Edipe («malheureuse » y a valeur analeptique, renvoyant à la connaissance qu'Edipe a des malheurs passés de la reine) qu'à celui du locuteur citant : "malheureuse " y acquiert une valeur proleptique, Li annonçant l'inceste qu'Edipe ignore. Même si l'on rajoutait un adverbe temporel dans l'énoncé cité, actualisé par rapport à la situation de LI (« il allait épouser demain cette malheureuse Jocaste», cela n'empêcherait pas que «cette malheureuse » puisse renvoyer au PDV de e2 :

(2a) Edipe fit part de ses projets aux citoyens de Thèbes : il allait épouser demain cette malheureuse Jocaste ainsi qu'il ne cessait de l'appeler, tant il avait été frappé par le récit de ses infortunes. Mais, malheureuse, Jocaste l'était doublement, en ajoutant l'horreur de l'inceste à ses souffrances passées!

Par conséquent, s'il n'y a dans un énoncé qu'un seul centre déictique, comme il appert des transpositions effectuées par le locuteur citant, en revanche, il est toujours possible qu'il y ait au moins deux 
centres modaux. Une telle déliaison n'est pas propre au DR, elle est fondamentale dans tous les énoncés dialogiques, à commencer par un énoncé aussi banal que (I), dont le locuteur premier est le narrateur, Li / E I entérinant par défaut les impatiences de Jeanne, énonciateur second (e2), Jeanne, auteur d'un PDV dans une "phrase sans parole" (Banfield I995). Il en va de même dans les mentions ironiques ou dans ou encore dans l'exemple bien connu de Ducrot, «l'ordre sera maintenu coûte que coûte », dans lequel LI / EI est en phase avec un énonciateur e2 faisant une promesse aux allocutaires «bons citoyens », et aussi avec un autre énonciateur e2' qui menace les allocutaires " contestataires ou mauvais citoyens ", laissant ouverte la question ${ }^{\mathrm{I}}$ de savoir si Li est plutôt du côté de la menace ou de la promesse, ou s'il considère que la réalisation de la menace est le garant de l'effectivité de sa promesse (ce qui n'entraîne pas les mêmes conséquences pratiques).

Ainsi, dans un énoncé monologique, le locuteur et l'énonciateur sont en syncrétisme. Il n'en va pas nécessairement de même, dans les énoncés dialogiques : car les énoncés marqués par le dialogisme interne ${ }^{2}$ ( «le deux de l'énonciation dans l'un (apparent) de l'énoncé », selon la formule de Bres et Verine 2002 : I62) peuvent certes se composer de deux (ou plus) locuteurs et énonciateurs, mais aussi d'un seul locuteur et de deux (ou plus) énonciateurs, dès lors qu'un PDV enchâssé non embrayé coréfère à un sujet de l'énoncé distinct de Li. Bref, un énoncé dialogique, s'il a toujours plusieurs énonciateurs, n'a pas nécessairement autant de locuteurs que d'énonciateurs.

Certes, la déliaison des actualisations déictique et modale présente l'inconvénient de laisser penser que la modalité n'aurait rien à voir avec la déixis : il n'en est rien, bien entendu, certains modes (indicatif, impératif, etc.), temps (présent, futur, conditionnel, etc.) ayant une valeur modale importante. Mais la conjonction des deux actualisations présente l'inconvénient rédhibitoire de sous-estimer les valeurs modales hors du système du je, ici, maintenant, ainsi que le remarque J.-M. Barbéris :

I. La question vaut tout autant pour le coénonciateur de l'énoncé.

2. Par opposition au dialogisme externe, interlocutif. 
En limitant la réflexion sur la subjectivité aux marques de la personne (pronoms personnels de première ou de deuxième personne), aux déterminants ou pronoms démonstratifs et possessifs (cet arbre, mon arbre, ça, le mien...), aux adverbes déictiques (ici/là / là-bas, hier / aujourd'hui/demain), on se prive de la possibilité de relier le sujet égotique, pleinement individualisé, aux autres formes d'apparition de la subjectivité, beaucoup plus discrètes et diffuses, mais effectives. Ici se trouve sans doute la limite des approches énonciatives en termes de marques [...] Car les plus claires (cf. les embrayeurs par exemple) deviennent aussi les arbres cachant la forêt, où se trouvent tous les autres modes de positionnement linguistique de la subjectivité. En particulier, le sujet expérientiel ne trouve aucune place dans ce tableau de la subjectivité, qui fonctionne en tout (le sujet est pleinement actualisé dans la marque de première personne, et dans l'ici spatial), ou rien (on postule bien une approche anthropologique et expérientielle, mais elle ne s'appuie sur aucune forme identifiable de subjectivité) (Barbéris, in Détrie, Siblot et Verine 200 I : 330).

C'est donc pour rétablir la balance en faveur d'un continent peu exploré que nous optons en faveur d'une déliaison théorique des actualisations, pour mieux rendre compte des dynamiques pratiques de la mise en scène énonciative dans un cadre radicalement dialogique. À charge de préciser le contenu de l'actualisation modale (et aussi, même si c'est un problème que nous n'aborderons pas ici, de clarifier ce qui relève de la modalité et de la modalisation, comme y invite Vion 2003).

On pourrait se demander, tout en partageant notre souci de distinction des actualisations déictique et modale, s'il est bien convenable de rattacher le centre de perspective de (I) à un énonciateur, comme nous le faisons (après Ducrot), ou s'il ne serait pas préférable de parler de "sujet de conscience» (Zribi-Hertz I996), d'《expérienceur» ou de « bilan ou de fil empathique» (Forest I 999). Le choix adopté ici est celui de l'économie (afin de ne pas multiplier les instances) et de l'explication la plus abstraite possible. Il est aussi de marquer la nature profondément énonciative des comptes rendus de perception (cf. infra 2.2.), ainsi que nous allons essayer de le montrer à présent, quand bien même la saisie de cette nature énonciative paradoxale invite à une redéfinition de l'énonciation, distinguant d'un côté ce qui relève classiquement de la prise en charge énonciative par un énonciateur en syncrétisme un locuteur, de l'autre d'une 
prise en charge par un énonciateur non locuteur. Il est enfin de souligner l'originalité d'un dialogisme interne dans lequel le sujet percevant « dialogue » plus ou moins intentionnellement avec lui-même, dans ses efforts cognitifs d'appréhension de l'objet (cf. infra, 2.3. et 3.).

\section{La représentation dialogique des comptes rendus de perception}

Venons-en à présent à l'analyse des comptes-rendus de perception. En règle générale, ces derniers, qui sont étudiés sérieusement sur le plan linguistique depuis deux décennies sont souvent comparés au DIL (Danon-Boileau I982), ou opposent le savoir du sujet de l'énonciation (locuteur primaire) à celui du sujet de l'énoncé (Vogeleer 1994). Ces analyses polyphoniques font ressortir la situation paradoxale dans laquelle ces verbes ont été longtemps maintenus, notamment dans les analyses narratologiques qui ont réduit les perceptions au rôle de dernier rempart de l'objectivité, à travers la focalisation " externe » ou la représentation de la focalisation zéro comme «absence de focalisation » (Rabatel 1997). Or les perceptions sont elles aussi passibles d'une analyse linguistique, puisque ces dernières sont " verbalisées ${ }^{\mathrm{I}}$ », (comme les pensées et les paroles) et que cette verbalisation révèle l'extrême implication des procès perceptuels et des procès mentaux (Franckel et Lebaud I990, Rabatel I998).

\section{I. Perceptions représentées et embryonnaires}

En (3), les perceptions représentées, c'est-à-dire qui comportent des IMP ( « il n'y avait personne dans les WC », « au fond, la surface de l'eau se ridait doucement») sont indiquées par le discordanciel énonciatif (Rosier I 999) entre le premier plan au PS et l'IMP, par les valeurs aspectuo-temporelles de ce dernier (cf. infra) et par le rapport sémantique entre la référenciation globale d'un événement perceptuel dans le premier plan (fait, perception appréhendée comme

I. «La linguistique n'a nul besoin de faire la distinction entre dire et pensée puisqu'en tant que représentée la pensée est verbalisée » (Nølke et Olsen 2000 : 87). 
un tout) et l'analyse de ses "parties » dans le deuxième plan ${ }^{\mathrm{I}}$. Le PDV est celui de l'enquêteur — quand bien même les perceptions représentées ne sont pas sous la rection d'un verbe de perception et d'un subordonnant, sous-entendus, à la différence de "il fit la lumière et constata qu'il n'y avait personne »-, en raison de la saillance de l'enquêteur, puisqu'il est, sur les plans sémantique, discursif et syntaxique un animé, déjà mentionné dans le discours, en position sujet, à l'origine d'un processus perceptuel.

(3) Il s'arrêta devant la porte, de façon à être protégé par le mur. Par l'interstice, il vit que les WC étaient plongés dans l'obscurité. Il essaya de deviner de quel côté se trouvait l'interrupteur et finit par estimer que ce devait être sur la gauche, puis alluma sa torche, braqua son arme et glissa la pointe de sa chaussure dans l'entrebâillement de la porte. Il donna un grand coup de pied et le battant s'ouvrit à la volée. Accroupi sur le seuil, il fouilla l'obscurité avec sa lampe, puis ses doigts rencontrèrent l'interrupteur et il fit la lumière - il n'y avait personne dans les WC. Il resta là, le cœur battant la chamade, avec toujours l'impression que quelqu'un se tenait là, tout près. Puis il entendit de nouveau un reniflement et comprit. Il s'avança et s'arrêta devant la cuvette en faïence. La réponse était sous les yeux : au fond, la surface de l'eau se ridait doucement. (Matti Yrjänä Joensuu Harjunpää et l'homme-oiseau, Gallimard 2000 : 22I)

Comme en (I), le PDV ne se limite pas à une dénotation des états du monde, puisque la subjectivité qui est à la source de la référenciation est en retour impliquée par cette dernière : c'est pourquoi le PDV ne dénote pas seulement des états du monde, il implique un faire penser et un faire agir de l'énonciateur et/ou des coénonciateurs. On voit ainsi l'enquêteur réagir face à un bruit jugé menaçant, puis, au terme de mouvements, perceptions et inférences, invalider ses impressions premières, avant de réajuster son comportement en fonction de la situation nouvelle.

On pourrait objecter que les perceptions représentées à l'IMP sont si proches du DIL que c'est du DIL. Wilmet I998 : 449 juge cette interprétation réductrice. En effet, si le PDV intrique le plus souvent (pour ne pas dire toujours) perceptions et pensées à travers les processus inférentiels qu'il active, comme il apparaît en (I) et

I. Cf. Rabatel I998 pour une analyse des paramètres énonciatifs du PDV. 
en (3), la réciproque n'est pas vraie, le DIL pouvant se limiter à des paroles ou à des pensées indépendamment de tout procès perceptuel (Rabatel 2003c). De plus, le PDV est loin de se limiter aux perceptions représentées à l'IMP, le compte rendu de perception peut également prendre la forme de perceptions embryonnaires (Rabatel 2000a, $2004 \mathrm{~b}^{\mathrm{I}}$ ) avec un verbe au passé simple. Par conséquent on trouve là de quoi contredire l'hypothèse (Rabatel I998, 200 Ia) selon laquelle le PDV serait une des formes du DR, à côté du DD, DI ou du DIL et en forte proximité avec ce dernier : en effet, «le» PDV peut s'apparenter tantôt à l'une ou à l'autre des formes du DR, comme on va le voir. La représentation du PDV comme une forme de DR à côté du DD, DI, DIL, ou du monologue intérieur ne s'explique que parce que l'analyse privilégie un PDV parmi d'autres (le PDV représenté), tout en survalorisant par ailleurs le rôle du DIL dans l'expression de la subjectivité.

Or il nous semble (à présent) plus conforme à la matérialité linguistique de partir de la diversité des structurations syntaxiquesénonciatives pour mieux rendre compte du dialogisme interne des énoncés comme des dynamiques par lesquelles se construit la subjectivité du sujet percevant/ pensant / parlant.

\subsection{Dialogisme des différents comptes rendus de perception}

Pour faire ressortir le dialogisme des comptes rendus de perception, on partira du fait qu'un contenu propositionnel approximativement similaire peut être exprimé en empruntant un compte rendu de parole, de pensée ou de perception. Cette possibilité se concrétise dans les choix de comptes rendus avec représentation directe, indirecte, indirecte libre, directe libre ou narrativisée (pour s'en tenir aux formes les plus conventionnelles du DR).

I. Le point de vue embryonnaire apparait avec des PS : il est dit embryonnaire, lorsque la perception n'est pas développée dans le deuxième plan par des IMP descriptifs / commentatifs. La dimension cognitive et axiologique y est de ce fait moindre que dans le PDV représenté, mais elle existe néanmoins, en fonction de la référenciation, tant du procès perceptuel (« aperçut » vs «scruta ») que de celle de l'objet perçu («vit une/la / sa voiture/guimbarde», etc. Pour une approche des marques entrant en compte, cf. Rabatel $2003 \mathrm{c}$. 
Par commodité, on s'appuiera ici sur des exemples fabriqués, plutôt que sur des exemples attestés. Il est certain que le passage d'exemples attestés à de telles constructions peut poser problème. Mais c'est le prix à payer pour neutraliser les différences lexicales, syntaxiques ou sémantiques de toute nature qui compliqueraient immanquablement l'observation, si l'on se basait sur des exemples attestés nécessairement disparates. Au demeurant, ces constructions renvoient à tant d'occurrences attestées ${ }^{\mathrm{I}}$ et sont si banales dans leur structuration syntaxique qu'elles échappent au reproche de «forgerie » de convenance.

Évidemment, quand nous disons que ces comptes rendus ont un contenu «approximativement similaire », nous n'ignorons pas que le choix d'un dire influe nécessairement sur le dit, comme on le verra à travers la question des gradients de réflexivité. Mais, au-delà de ces différences, ce que les contenus propositionnels ont d'essentiel en commun réside dans le fait que tous expriment l'altérité énonciative, qui existe in nucce dans les comptes rendus de perception et qui s'extériorise dans les comptes rendus de paroles et de pensées.

Les comptes rendus de perception dont le dialogisme est le plus difficile à repérer sont les comptes rendus direct, direct libre et narrativisé, en raison du faible marquage de l'altérité énonciative ${ }^{2}$.

(4) Compte rendu direct de parole : Pierre s'approcha de la fenêtre, regarda le convoi funèbre et dit : «l'assistance est nombreuse »

(5) Compte rendu direct de pensée : Pierre s'approcha de la fenêtre, regarda le convoi funèbre et se dit : «l'assistance est nombreuse »

(6) Compte rendu direct de perception : Pierre s'approcha de la fenêtre et regarda le convoi funèbre : l'assistance était nombreuse / l'assistance est nombreuse.

Comme on l'a vu en (3) les marques du PDV, dans les italiques de (6) correspondent au PDV de e2, Pierre, l'énonciateur saillant. Syntaxiquement, (6) s'apparente à (4) et (5), car il repose sur un « rapport» direct, en l'absence de lien hypotaxique entre le verbum

I. Le lecteur trouvera des exemples attestés de ce genre dans Rabatel 2003a : 70-7I.

2. C'est aussi le cas pour les comptes rendus indirects libres, mais leur fréquence, tout comme l'importance des traces des procès mentaux, les rendent plus aisément repérables. 
sentiendi et le complément d'objet. Néanmoins, il en diffère notablement, en l'absence des marques traditionnelles de compte rendu direct, guillemets, deux points et verbum dicendi. Ces absences ne sont toutefois pas de nature à enrayer le dialogisme du PDV, lorsqu'il emprunte un rapport direct.

L'absence de guillemets paraît en effet un critère de portée relative si l'on pense que Rosier donne pas moins de I 5 autres marques annonciatrices de DD - avec incise, avec verbum dicendi + virgule, avec verbum dicendi + deux points sans guillemets, avec italiques (avec ou sans guillemets), avec tirets, avec parenthèses + guillemets, etc. (Rosier I 999 : 208-209). Il en ressort que le compte rendu de perception sans guillemets n'est pas unique, cette absence (certes rare) étant compensée par d'autres marques, notamment le verbum dicendi, à la semblance, en (6), de la mention explicite d'un verbe de perception dont le sujet est Pierre. Il n'en reste pas moins que leur absence complique le bornage du compte rendu de perception. Au demeurant, la relativité du critère des guillemets repose surtout sur le fait que ce critère vaut pour l'écrit (et ce depuis quelques siècles seulement) mais pas pour l'oral. On ajoutera ici une expérience personnelle maintes fois vérifiée lors de la lecture à voix haute des comptes rendus de perception, y compris chez des lecteurs qui ne sont pas au fait de nos analyses : il se produit des changements de débit, de mélodie et d'intensité analogues à ce qu'on observe dans le DIL, tout particulièrement lorsque le compte rendu de perception est introduit par un présentatif (Rabatel 2000 b et 200Id). Même s'il faut se méfier d'observations qui n'ont pas fait encore l'objet de mesures acoustiques précises, il semble que l'intuition soit à prendre en considération dans l'argumentaire en faveur de la nature dialogique des perceptions.

La présence du verbe de perception et de son sujet n'équivaut toutefois pas, sur le plan sémantique, à un verbum dicendi ou putandi : ici, il faut convenir que la perception alimente des processus inférentiels de toute sorte, qui sont rapportés au sujet de l'énoncé. C'est ce qui se vérifierait en (6a) si l'on supprimait le verbe de perception :

(6a) Pierre s'approcha de la fenêtre : l'assistance était nombreuse / l'assistance est nombreuse. 
Du fait de la saillance de Pierre, du fait des marques listées auparavant, le lecteur infère, en vertu de sa connaissance du monde, que i) Pierre s'est approché de la fenêtre pour regarder et que ii) ce qui est vu est le résultat de la perception de Pierre, en vertu des marques co-textuelles.

Quant aux motivations de Pierre, elles ne sont pas mentionnées dans le co-texte, à la différence de (I) où les inférences activées par le groupe participial «ayant fini ses malles » indiquent une volonté de sortir contrecarrée (cf. "mais ») par la pluie, et le fort dépit qui en résulte, ainsi que le signale le sémantisme du verbe, renforcé par la forme négative, "ne cessait pas ». Il est à noter que le PDV resterait celui de Jeanne si l'on supprimait «mais» ou si l'on remplaçait « ne cessait pas» par « il pleuvait»: mais il serait alors réduit à sa plus simple expression, comme en (6) et en (6a), c'est-à-dire à l'expression d'une altérité énonciative, sans que cette altérité ne soit davantage précisée, par défaut d'information.

De ce point de vue, la différence entre compte rendu de perception et comptes rendus de paroles ou de pensée est significative. L'hypothèse, sur laquelle on reviendra in fine, est que cette différence dans le marquage énonciatif explicite ou implicite (et le coût inférentiel qui en résulte) renvoie à la dimension pré-réflexive des perceptions. Ainsi, au-delà des différences, ce qui unit (6) à (4) et (5), c'est la dimension dialogique, centrée sur un rapport de soi à autrui (rapports entre LI/EI et $12 / \mathrm{e} 2$ ou e2) ou de soi (idem) à soi (ipse), par exemple du soi percevant dans son état pré-réflexif au soi réflexif. Et il n'est pas sans intérêt de remarquer que ce dialogisme interne du soi peut sans peine s'exprimer par le compte rendu de pensée ou de parole, notamment dans le monologue intérieur...

La présence de l'IMP dans une des variantes de (6), concurremment au présent, soulève d'autres difficultés. Comme on l'a dit, l'IMP apparente ce compte rendu direct au DIL ${ }^{1}$. Cet IMP indique un espace mental autre dans le discours du locuteur : Bres 2003 : I 6 évoque à son propos un "marqueur d'altérité énonciative». En effet, sa valeur aspectuo-temporelle suppose « une dissociation de la source énonciative, mettant entre parenthèse le hic et nunc de

I. L'argument est d'ailleurs critiquable en ce qu'il survalorise les occurrences de DIL avec troisième personne + IMP, alors qu'il existe des DIL avec première personne + présent de l'indicatif ou du « conditionnel». 
l'énonciateur primaire au profit d'un ancrage sur le repère secondaire » (Mellet 2003 : 90), l'aspect sécant de l'événement passé invitant à saisir le procès de l'intérieur. En vertu de quoi la perception est à la fois ancrée dans le passé de la diégèse (ce qui signifie que l'assistance était nombreuse avant que Pierre ne s'approche de la fenêtre) et aussi considérée à travers le prisme subjectif de Pierre ${ }^{\mathrm{I}}$. Le choix d'un présent (qui rapproche le compte rendu de perception du DDL, alors que l'IMP le tire vers le DIL) indique un commentaire sur le vif, dans le prolongement quasi immédiat d'une saisie perceptuelle rapportée au nunc de l'écriture et de la lecture : il ne s'agit pas d'un présent déictique, mais d'« une pure adhésion au déroulement procédural de la représentation mentale des événements successifs » (Barbéris et Bres 2002 : I02).

Bref, le PDV, sous la forme du rapport direct de perception, est aux confins de la problématique du DR, mais au cœur de celle du dialogisme, même si son marquage obéit à des spécificités qui renvoient, en dernière instance, à la dialectique des relations entre réflexivité et pré-réflexivité ${ }^{2}$. On peut en dire autant des comptes rendus direct libre et narrativisé :

(7) Compte rendu direct libre de parole : Pierre s'approcha de la fenêtre, regarda le convoi funèbre. Que l'assistance est nombreuse! Cette remarque fut accompagnée d'un sourire.

(8) Compte rendu direct libre de pensée : Pierre s'approcha de la fenêtre, regarda le convoi funèbre. L'assistance est nombreuse. Cette pensée fugace le réconforta.

(9) Compte rendu direct libre de perception : Pierre s'approcha de la fenêtre et regarda le convoi funèbre : assistance nombreuse, deux cents personnes environ/Pierre s'approcha de la fenêtre et regarda le convoi funèbre. Spectacle réconfortant d'une assistance nombreuse, deux cents personnes environ.

I. Et par surcroît du locuteur premier, qui, ici, ne manifeste pas de distance, ce qui autorise le lecteur à entériner comme vrai l'événement représenté. On notera qu'à défaut de sujet saillant, le mécanisme de représentation des perceptions reste identique, et la perception représentée est attribuée à LI / EI.

2. Banfield I 995 293-3I3 nomme non-réflexif ce que nous appelons pré-réflexif : sa dénomination renvoie au binarisme structuraliste, alors que nous privilégions une approche dynamique/dialectique du sujet, qui se traduit linguistiquement par des continuums (Rabatel 200Ic : 89-93). 
(Io) Compte rendu narrativisé de parole (= discours narrativisé) : Pierre s'approcha de la fenêtre; là, s'adressant à Charles pendant de longues minutes, il se félicita de l'importance du convoi funèbre.

(I I) Compte rendu narrativisé de pensée (= psycho-récit) : Pierre, s'approcha de la fenêtre; là, il imagina les bonnes raisons que Marc allèguerait pour n'avoir pas rejoint l'important convoi funèbre.

(I2) Compte rendu narrativisé de perception (= PDV embryonnaire) : Pierre, s'approcha de la fenêtre et s'amusa à voir un Marc hésitant avant de prendre part à la nombreuse assistance du convoi funèbre.

La parenté des relations hypotaxiques de (4)-(6) et (7)-(9) explique que les énoncés en italiques puissent légitimement être interprétés, selon les données co-textuelles (en gras), comme un compte rendu direct de perception, ou, du fait de l'absence de données contextuelles, comme un compte rendu direct libre de parole ou de pensée. De même, en (9), si « spectacle» oriente l'interprétation vers un compte rendu direct de perception, en revanche, "assistance nombreuse, deux cents personnes environ » dénote une perception intentionnelle, une activité cognitive pouvant s'interpréter comme compte rendu de pensée, voire comme compte rendu de parole, si l'on considère que le mouvement de la pensée est (infra)verbalisé. Cette hésitation n'affaiblit pas l'analyse, elle exhibe au contraire les continuums existant sur les plans phénoménologique et cognitif entre perception, pensée et parole (Damasio I994, I999), tout comme sur le plan linguistique (Rabatel 200 Ic : 93, 2003a : 72).

Au demeurant, les autres comptes rendus de perception, indirect, indirect libre ne posent guère de problèmes sur le plan syntaxique et énonciatif, comme on peut le vérifier avec les exemples suivants :

(I3) Compte rendu indirect de parole : Pierre s'approcha de la fenêtre, regarda le convoi funèbre et dit que l'assistance était nombreuse.

(I4) Compte rendu indirect de pensée ${ }^{\mathrm{I}}$ : Pierre s'approcha de la fenêtre, regarda le convoi funèbre et pensa que l'assistance était nombreuse.

I. Cette possibilité est très fréquente dans les discours rapportés oraux comme l'a montré une récente analyse de Marnette 2002 : 21 8-220, selon laquelle, en français parlé, les pensées ne sont pas rapportées au DIL (seulement $4 \%$ des formes répertoriées dans les corpus du GARS inventoriés), mais majoritairement au DI. 
(I5) Compte rendu indirect de perception: Pierre s'approcha de la fenêtre, regarda le convoi funèbre et remarqua que l'assistance était nombreuse. / Pierre vit que l'assistance était nombreuse.

(i6) Compte rendu indirect libre de parole : Pierre s'approcha de la fenêtre, regarda le convoi funèbre. Il attira l'attention de Jean. L'assistance était nombreuse!

(17) Compte rendu indirect libre de pensée : Pierre s'approcha de la fenêtre, regarda le convoi funèbre. Que l'assistance était nombreuse!

(I8) Compte rendu indirect libre de perception : Pierre s'approcha de la fenêtre, regarda le convoi. L'assistance était vraiment nombreuse!

Ainsi, les divers comptes rendus de perception expriment plus ou moins nettement une altérité énonciative entre sujet de l'énoncé et sujet de l'énonciation, altérité qui traverse les sujets eux-mêmes. Une telle complexité invite à s'intéresser aux marques susceptibles de mettre de l'ordre dans l'hétérogénéité énonciative, ce qui aura pour effet de clarifier les relations que le soi entretient avec d'autres ego, mais encore les relations que le soi entretient avec lui-même.

\subsection{Rôle de l'actualisation modale dans le marquage externe et interne des comptes rendus de perception}

Ce continuum est en effet marqué par la fréquence des phénomènes d'hétérogénéité énonciative. Si les marques ouvrantes et fermantes sont aisément repérables dans le DD, elles le sont moins dans le DI (on sait où il commence, mais pas toujours où il s'arrête), moins encore dans le DIL, le DDL, le discours narrativisé. Semblablement, les marqueurs de portée, tels «d'après / selon / à en croire Pierre, l'assistance était nombreuse », etc., indiquent l'ouverture d'un espace énonciatif autre dans le discours du locuteur, sans toujours préciser où s'arrête le dire d'autrui. Et le problème est encore plus complexe lorsque le PDV se limite à l'inclusion de subjectivèmes en l'absence de bornes ouvrante et fermante. C'est pourquoi il est crucial, pour l'interprétation des PDV dans des "phrases sans parole » de lister quelques-unes des marques externes (de bornage) et internes (d'actualisation modale) indiquant l'ouverture, ou, à défaut, la trace d'un espace énonciatif autre dans le dire d'ego : 
Marques externes : les marques ouvrantes ou fermantes sont indiquées par un procès de perception (un verbe, le plus souvent, mais aussi un nom, comme en (9)) et un sujet percevant afférent; en l'absence de ces marques, le procès de perception peut être inféré d'un verbe de mouvement; l'opposition des formes globales et sécantes joue un rôle ouvrant (passage du premier plan au second plan) ou fermant (passage du second plan au premier plan) à l'instar de ce qui se passe avec le DIL (Vuillaume $2000^{\mathrm{I}}$ ).

Marques internes: plus les marques externes de bornage énonciatif sont ténues; plus ce sont les marques internes d'actualisation modale qui jouent le rôle de signaux d'altérité énonciative, et qui ont le mérite d'incarner les instances à l'origine des PDV.

De nombreux éléments lexicaux sont susceptibles de jouer ce rôle de marque interne. Ainsi, au plan de la cohésion nominale, des dénominations lexicales comportant des jugements de valeur appréciatifs ou dépréciatifs ( I9) ou les phénomènes d'actualisation du nom (20) :

(19) La populace était nombreuse / grouillait.

(20) Une / la / cette foule inspirait le respect.

Au plan de la cohésion logique, la valeur énonciative-argumentative des connecteurs et des marqueurs temporels et spatiaux, des intensifs jouent ce rôle de marque interne (Rabatel I999, 200 Ib) :

(2I) Pierre s'approcha de la fenêtre et regarda le convoi funèbre : mais, l'assistance pourtant si nombreuse, ne se mettait toujours pas en branle

(22) Pierre s'approcha de la fenêtre et regarda le convoi funèbre : à sa droite / cependant / maintenant, l'assistance, nombreuse, se mettait en branle

Au plan verbal, les choix aspectuo-temporels (cf. supra), le sémantisme des verbes ( «l'assistance venait s'engouffrer sur la place», les

I. Les alternances PS $\rightarrow$ IMP $\rightarrow$ PS ne signifient pas nécessairement la fin d'un PDV et le retour au récit. Comme on l'a montré dans Rabatel 2003b, 2004b, elles indiquent aussi le passage d'un PDV représenté à un PDV embryonnaire (ou inversement), autrement dit des degrés dans l'appréhension réflexive des percepts par e2. 
présentatifs (Rabatel 2000 b et $200 \mathrm{Id}$ ), etc. contribuent également à l'actualisation modale.

Sur le plan syntaxique, la plupart des marques de dialogisme jouent le rôle de marque interne et tirent le compte rendu de perception vers un compte rendu de parole ou de pensée, et confirment que le PDV est celui de Pierre, instancié comme 12/e2 à la source des perceptions, soit par une attribution explicite (cf. (26) : « ce qui le frappa »), soit par défaut, les procès mentaux, ouvertement dialogiques étant a fortiori encore plus facilement attribués à l'énonciateur saillant que des procès perceptuels au dialogisme ténu. Il en va ainsi pour l'interrogation (23), la question rhétorique (24), la présupposition ou la négation (25), la phrase clivée (focalisation, topicalisation) (26), la concession (27), l'opposition (28), la confirmation (29), la rectification (30), le renchérissement (3I) :

(23) Pierre s'approcha de la fenêtre; le convoi funèbre serait-il important? Oui, l'assistance était nombreuse!

(24) Pierre s'approcha de la fenêtre et regarda le convoi funèbre : vit-on jamais assistance si nombreuse?

(25) Pierre s'approcha de la fenêtre et regarda le convoi funèbre : l'assistance n'était pas ridicule

(26) Pierre s'approcha de la fenêtre et regarda le convoi funèbre : ce qui le frappa, c'est la nombreuse assistance / cette assistance si nombreuse, quel choc!

(27) Pierre s'approcha de la fenêtre et regarda le convoi funèbre : même si l'assistance était peu nombreuse, elle était déterminée

(28) Pierre s'approcha de la fenêtre et regarda le convoi funèbre : l'assistance n'était pas ridicule, elle était impressionnante

(29) Pierre s'approcha de la fenêtre et regarda le convoi funèbre : oui, l'assistance était nombreuse

(30) Pierre s'approcha de la fenêtre et regarda le convoi funèbre : l'assistance, enfin le carré des personnes présentes, était assez nombreuse

(3I) Pierre s'approcha de la fenêtre et regarda le convoi funèbre : l'assistance, en fait, une vraie foule, était impressionnante

Ces marques dialogiques explicitent la dimension responsive sous-jacente in nucce dans (6) : par exemple, le compte-rendu perceptuel dialogique de (24) est une réponse à une objection implicite 
présupposée, (25) et (27) sont une réponse à une objection antérieure (ou en tout cas anticipent sur cette objection), et ainsi de suite. Le sujet de l'énoncé ${ }^{\mathrm{I}}$ serait également le centre des actualisations modales avec ces autres marques de dialogisme que sont la reprise-écho, le détournement, l'ironie, le conditionnel, la modalisation autonymique, etc., quand bien même ces marques indiqueraient de surcroît une distanciation de Li envers $l 2$ / e2.

Ainsi, en fonction du dialogisme des marques linguistiques entrant en jeu dans la référenciation des perceptions, ces dernières dénotent des procès perceptuels plus ou moins intentionnels ${ }^{2}$ et plus ou moins chargés de dimensions épistémiques et axiologiques, au point que la saturation des marques dialogiques brouille la (trop commode?) frontière entre perception d'un côté, pensée ou parole de l'autre. Or c'est précisément ce passage de la frontière qui requiert notre intérêt. Ces nouveaux exemples de continuum entre perceptions, pensées et paroles méritent d'être appréhendés à présent à la lumière de nos hypothèses sur la pré-réflexivité des perceptions, et la réflexivité des paroles ou des pensées. Nous le ferons en confrontant nos hypothèses avec celles de Barbéris sur la subjectivité en idem et en ipse.

\section{3. (Pré-)réflexivité des perceptions et subjectivité en idem lipse}

L'importance des marques précédentes dans le marquage de la subjectivité autres que celles entées sur le je, ici, maintenant, donne " une épaisseur concrète à la transition entre langue et discours » (Barbéris 200I : 328). En effet, les marques d'actualisation modale ne se contentent pas de lever des ambiguïtés énonciatives, elles jouent encore un rôle dans la construction du sujet (percevant), selon leur nature réflexive ou pré-réflexive, nature elle-même direc-

I. Certes, il est vraisemblable que Li soit en consonance avec les procès mentaux exprimés, ne serait-ce que par défaut, mais cette consonance vient se surajouter à la présence empathique du sujet de l'énoncé, qui est un centre modal à côté de cet autre centre modal qu'est le locuteur citant.

2. D'autres facteurs entrent bien évidemment en compte, notamment le sémantisme des verbes de perception, les données co-textuelles (cf. en (I) et en (3)), ou encore le choix de telle ou telle structure syntaxique (Rabatel I998 : 20-22. 
tement dépendante, comme on va le voir à présent, de la densité de l'actualisation modale entrant dans la référenciation des percepts.

On a parlé plus haut de continuum entre perception, pensée et parole. En fait, ce continuum fonctionne également à l'intérieur des perceptions, qui ne sont pas un bloc monolithique, ces dernières relevant de formes d'expression pré-réflexives ou réflexives. Cette opposition graduelle s'entend d'abord en un sens énonciatif : les formes pleinement actualisées, sur un plan déictique (DD) sont réflexives et les formes faiblement actualisées, pré-réflexives (DIL, PDV), car la subjectivité et l'activité cognitive qui l'accompagne se laissent plus difficilement repérer dans les énoncés délocutés, où l'on entend moins la voix d'un locuteur qu'on ne repère des manières de voir d'un énonciateur. Cette opposition a ensuite un contenu sémantique : la réflexivité renvoie à une pensée consciente, rationnelle, élaborée tandis que la pré-réflexivité exprime une forme de pensée préconsciente, pré-rationnelle, fugace, non élaborée par la distance critique que permet le discours. Ces dichotomies reposent sur la représentation commune qui associe la pensée réfléchie et l'objectivation des marques de la parole (cf. Rabatel 2003b : I 6-28 pour un examen plus détaillé).

Ainsi, les perceptions sont conventionnellement moins réflexives que les paroles ou les pensées. Mais cette donnée peut être contrebalancée par le nombre et la nature des marques d'actualisation modale. Ainsi, si le DD est en principe plus réflexif que le $\mathrm{DN}$ ou le PDV, en revanche, un DN ou un PDV comprenant de nombreuses traces d'actualisation modale deviennent aussi réflexifs qu'un DD faiblement « mimétique».

L'actualisation modale, dont on a listé un certain nombre (non exhaustif) de marques, exprime ainsi la subjectivité sous un double aspect, en jouant un rôle expressif et cognitif indiquant les efforts d'appréhension des objets par le sujet (ce qu'on a appelé ailleurs un mimétisme cognitif, Rabatel 2003b : 22 et 2004a). Les interrogatives, les exclamatives, les interjections sont à la fois des marques expressives, indiquant les réactions du sujet, mais aussi cognitives, témoignant des efforts d'ajustement du sujet face à son environnement. Il en va de même pour les hésitations, répétitions, pour les modaux, les adverbes modalisateurs, les dénominations ou qualifications évaluatives ou axiologiques, susceptibles de scander les mouvements de la 
pensée pour se rapprocher de la compréhension des objets. Chacun des exemples (4)-(I8) pourrait se voir enrichi (ou lesté, question... de point de vue) par les marques citées en (I9)-(3I).

La possibilité pour un même contenu propositionnel de prendre la forme d'un compte rendu de parole, de pensée ou de perception et d'être saturé (ou non) de marques d'actualisation modale participe de la dynamique de la subjectivité. Barbéris trace un parallèle entre le cinétisme à l'œuvre dans l'actualisation temporelle et nominale et la dynamique de la subjectivité en idem/ipse : en effet, si ego correspond au troisième stade de l'expression de la subjectivité, objectivant pleinement le sujet et l'individualisant comme instance spécifique (ipse), il existe en amont de lui des stades liminaires qui reposent sur des «schémas d'expérience partagée», qui peuvent être interprétés en termes de pré-réflexivité, notamment pour le stade liminaire, dans lequel «le sujet est une instance anonyme engagée dans la perception du monde» :

C'est à lui qu'on rapportera les schémas anthropologiques de type actantiel et les schémas de participation donnant lieu aux phénomènes d'empathie (au sens de Forest 1999). Ce centre d'expérience implicite est également celui que Franckel et Lebaud ont conçu comme source de point de vue dans un verbe comme voir (1990). Il se conçoit également comme engagement dans l'expérience. On rejoint ainsi la notion de gestalt expérientielle, proposée pour rendre compte des schémas sensori-moteurs qui sous-tendent les conceptions des sujets parlants (Barbéris 200I : 332).

Les comptes rendus de perception donnent souvent, en effet, l'apparence de renvoyer à une instance anonyme, anonymat qui facilite l'impression d'expérience partagée, et, par là, d'objectivité. Le sujet y est présent/ absent, présent par des marques (plus ou moins nombreuses) d'actualisation modale, absent sur le plan de l'actualisation déictique. On retrouve, à travers l'intensification de l'actualisation modale, souvent corrélée à l'actualisation déictique, les échos des phases 2 et 3 de la construction du sujet représentées dans le tableau suivant : 


\begin{tabular}{|c|c|c|}
\hline $\begin{array}{l}\text { SUJET EN } M \hat{E} M E: \\
\text { même de similitude } \\
\text { image de réalité virtuelle }\end{array}$ & $\begin{array}{l}\text { même analogique } \\
\text { image de réalité émergente }\end{array}$ & $\begin{array}{l}\text { SUJET EN SOI-MÊEE } \\
\text { ipse } \\
\text { image de réalité achevée }\end{array}$ \\
\hline $\begin{array}{l}\text { position : même } \\
\text { subjectivité implicite, } \\
\text { procédurale }\end{array}$ & $\begin{array}{c}\text { dialectique même / autre } \\
\text { intersubjectivité }\end{array}$ & $\begin{array}{l}\text { même vs autre } \\
\text { subjectivité explicite } \\
\text { (ipséité) }\end{array}$ \\
\hline $\begin{array}{l}\text { format praxéologique } \\
\text { exemples : } \\
\text { prépositions spatiales } \\
\text { phénomènes empathiques }\end{array}$ & $\begin{array}{l}\text { format dialogique } \\
\text { exemples : } \\
\text { déictique là, pronom on } \\
\text { phénomènes dialogiques }\end{array}$ & $\begin{array}{l}\text { format personnel } \\
\text { exemples : } \\
\text { embrayeurs je, ici } \\
\quad \text { maintenant }\end{array}$ \\
\hline
\end{tabular}

(Barbéris 200I : 333-4)

Mais, comme on l'a montré, il est toujours possible que le contenu propositionnel soit référé d'une telle façon que le sujet s'inscrive dans ses comptes rendus, à travers une construction dialogique, intersubjective de ses expériences perceptuelles, telle que la référenciation des perceptions construise une mimèsis du sujet et de l'objet (Rabatel 200 I b : 57). Ici, dialogisme de la langue et dialectique de la construction du sujet empruntent aux mêmes mécanismes.

Compte tenu de la variabilité des marques d'actualisation nominale et verbale en jeu dans la référenciation, il s'ensuit que les perceptions ne sont pas nécessairement cantonnées dans le «même de similitude », sauf à être faiblement actualisées, et qu'elles peuvent se retrouver dans le stade 2 du "même analogique », lorsqu'elles témoignent du dialogisme à travers les phénomènes de dissensus et de consensus et dans le stade 3, couplées avec le je, ici, maintenant. On peut même risquer l'hypothèse que, dans les contextes à la troisième personne et au passé, la densité et la variété des marques d'actualisation modale expriment un sujet incarné (à tout le moins impliqué) et réfléchi et viennent compenser l'absence de l'actualisation déictique (quand bien même cette forme de réflexivité est différente de la réflexivité ancrée de le hic et nunc).

La déliaison théorique des actualisations déictique et modale permet ainsi, outre sa contribution à une représentation moins abstraite des énonciateurs, de ne pas alimenter la reproduction à l'identique du couple infernal objectivité/ subjectivité, puisque la subjectivité qui est à la source de la référenciation est en retour impliquée par cette dernière : c'est pourquoi le PDV ne dénote pas seulement des états du monde, il implique un faire penser et un faire agir de 
l'énonciateur et/ou des coénonciateurs. Du même coup, cette dialectique invite à reprendre le chantier de l'engagement et de la prise en charge de PDV dans des énoncés «qui ne sont pas des paroles ».

\section{Références bibliographiques}

Banfield A. $\quad$ 1995, Phrases sans parole. Paris : Le Seuil.

Barbéris J.-M. 200I, Articles «subjectivité », «subjectivité dans le langage », "subjectivité en même vs en soi-même », in Détrie, C. Siblot, P. et Verine, B. 200 I.

Barbéris J.-M et Bres J.

2002, «Analyse textuelle de l'incipit», in Roulet, E. et Burger, M. (eds) Les modèles du discours au défi d'un "dialogue romanesque»: l'incipit du roman de $R$. Pinget, Le Libera. Nancy, PUN : 83-I23.

Bres J. 2003, «Mais oui, il était un joli temps du passé comme les autres, le petit imparfait hypocoristique », Langue française I38, I I I-I 25 .

Bres J. et Verine B. 2002, «Le bruissement des voix dans le discours : dialogisme et discours rapporté », Faits de Langues i 9 , I 59-I 69 .

Charaudeau P. et Maingueneau D. 2002, Dictionnaire d'analyse du discours. Paris : Le Seuil.

Damasio A. $\quad 1999$ (1994), L'erreur de Descartes. Paris : Odile Jacob.

Damasio A. I999, Le sentiment même de soi. Corps, émotion, conscience. Paris : Odile Jacob.

Danon-Boileau L. 1982, Produire le fictif. Paris : Klincksieck.

Détrie C., Siblot P. et Verine B.

200I, Termes et concepts pour l'analyse du discours. Paris : Champion.

Ducrot O. $\quad$ I980, «Analyses pragmatiques », Communications 32, I I -60.

Ducrot O. $\quad$ I984, Le dire et le dit. Paris : Minuit.

Ducrot O. I I993, «À quoi sert le concept de modalité ?» in Dittmar, N. et Reich, A. (eds) Modalité et acquisition des Langues. Berlin : Walter de Gruyter, I I-I 29. 
Forest R. I 999, Empathie et linguistique. Paris : Presses Universitaires de France.

Franckel J.-J. \& Lebaud D.

I990, Les figures du sujet. À propos des verbes de percep-

Guillemin-Flescher J.

tion, sentiment, connaissance. Gap, Paris : Ophrys.

2003, «Sujet énonciateur et sujet de l'énoncé dans la relation d'interlocution fictive », in Le sujet, Merle, J.-M. (ed) I83-I92. Gap, Paris : Ophrys.

Marnette S. 2002, «Étudier les pensées rapportées en français : mission impossible?». Faits de Langues I9, 2 I-220.

Mellet S. 2003, «Imparfaits en contexte : les conditions de la causalité inférée », Langue française I38, 86-96.

Nølke H. et Olsen M.

2000, «Polyphonie : théorie et terminologie », Polyphonie-linguistique et littéraire 2, 45-I7I. Université de Roskilde, Danemark.

Philippe G. 2002, «L'appareil formel de l'effacement énonciatif et la pragmatique des textes sans locuteur » in Pragmatique et analyse des textes, Amossy R. (ed), I7-34. Université de Tel-Aviv.

Rabatel A. $\quad$ I997, Une histoire du point de vue. Klincksieck, CELTED, Metz : Université de Metz.

Rabatel A. $\quad$ I998, La construction textuelle du point de vue. Lausanne, Paris : Delachaux et Niestlé.

Rabatel A. $\quad$ I999, «Mais dans les énoncés narratifs : un embrayeur du point de vue et un organisateur textuel », Le Français Moderne LXVII, I, 49-6o.

Rabatel A. 2000a, «Un, deux, trois points de vue? Pour une approche unifiante des points de vue narratifs et discursif », La Lecture Littéraire 4, I95-254.

Rabatel A. $\quad 2000 b$, "Valeurs représentative et énonciative du "présentatif" c'est et marquage du point de vue », Langue Française I28, 52-73.

Rabatel A. 200Ia, «Le point de vue et l'organisation du texte », Quelles grammaires enseigner à l'école et au collège? Discours, genres, textes, phrases. Garcia-Debanc, C. et alii (eds), 227-240. Toulouse : Delagrave-CRDP de Midi-Pyrénées. 
Rabatel A. 200Ib, « La valeur délibérative des connecteurs et marqueurs temporels mais, cependant, maintenant, alors, et dans l'embrayage du point de vue. Propositions en faveur d'un continuum argumentativo-temporel », Romanische Forschungen II3, 2, I 53-I 70.

Rabatel A. 200Ic, «Les représentations de la parole intérieure. Monologue intérieur, discours direct et indirect libres, point de vue » Langue Française I32, 72-95.

Rabatel A. 200Id, «Valeurs énonciative et représentative des "présentatifs" c'est, il y a, voici/voilà : effet point de vue et argumentativité indirecte du récit », Revue de Sémantique et Pragmatique 9/Io, 43-74. Orléans : Presses Universitaires d'Orléans.

Rabatel A. 2003a, «Les verbes de perception en contexte d'effacement énonciatif : du point de vue représenté aux discours représentés ", Travaux de linguistique 46-I, 49-88.

Rabatel A. $\quad 2003$ b, « La (pré)-réflexivité dans les comptes rendus de perception, de paroles ou de pensée : la question du mimétisme dans les discours représentés », in Polyphonie linguistique et littéraire, 7, I-3 I. Working papers. Université de Roskilde.

Rabatel A. 2003c, «Une lecture énonciative des valeurs aspectuotemporelles et commentatives de l'imparfait dans les suites PS + IMP : point de vue du locuteur ou de l'énonciateur », Journal of French Language Studies, I3-3.

Rabatel A. 2004a, " Entre usage et mention : la notion de représentation dans les discours représentés », L'apport de l'analyse du discours dans les études littéraires, Amossy, R. et Maingueneau, D. (eds), Io9-I I9. Toulouse : Presses Universitaires du Mirail.

Rabatel A. 2004b, « Les formes d'expression de la pré-réflexivité dans le discours indirect libre et dans les points de vue représentés ou embryonnaires ». Bulletin de la Société de Stylistique anglaise, 24 (sous presse).

Rosier L. I999, Le discours rapporté. Bruxelles : Duculot.

Vion R. 200I, " "Effacement énonciatif” et stratégies discursives ", in De la syntaxe à la narratologie énonciative, 
De Mattia, M. et Joly, A. (eds), 33 I-354. Gap, Paris : Ophrys.

Vion R. 2003, « Le concept de modalisation : vers une théorie linguistique des modalisateurs et de la modalité », Travaux du cercle linguistique d'Aix-en-Provence, I 8, 209229.

Vogeleer S. $\quad$ I994, «L'accès perceptuel à l'information : à propos des expressions un homme arrive/on voit arriver un homme », Langue française I02, 69-83.

Vuillaume M. 2000, «Le style indirect libre et ses contextes », Cahiers Chronos 5, I07-I30.

Wilmet M. I I998, Grammaire critique du français. Paris, Bruxelles : Hachette, Duculot.

Zribi-Hertz A. I996, L'anaphore et les pronoms. Villeneuve d'Ascq : Presses Universitaires du Septentrion. 